FTPI-MINN-10/17, UMN-TH-2909/10

July 2

\title{
Understanding Confinement in QCD: Elements of a Big Picture
}

\author{
Mikhail Shifman \\ William I. Fine Theoretical Physics Institute, University of Minnesota, \\ Minneapolis, MN 55455, USA
}

\begin{abstract}
I give a brief review of advances in the strong interaction theory. This talk was delivered at the Conference in honor of Murray Gell-Mann's 80th birthday, 24-26 February 2010, Singapore.
\end{abstract}




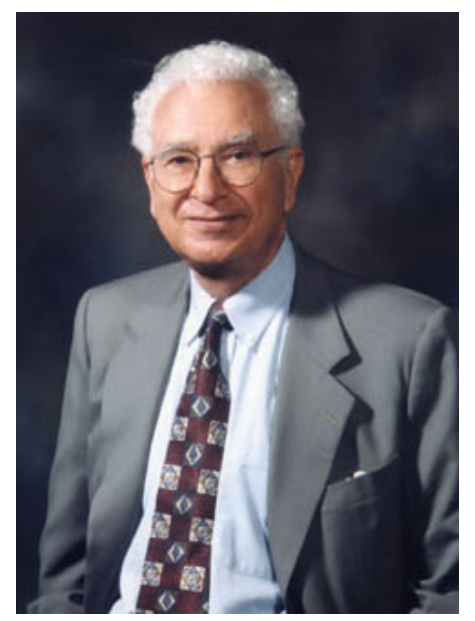

Figure 1: Everybody knows that this is Murray Gell-Mann.

\section{Introduction}

In the early 1970s, when quantum chromodynamics (QCD) was born, I was just in the beginning of my career in theoretical physics. My teachers at ITEP 1 tried to convey to me a number of "commandments" which were intended for guidance in my future scientific life. One of them was: always listen to what Gell-Mann says because he has a direct line to God. I always did. Gell-Mann was one of the discoverers of QCD who opened a whole new world. Unlike many recent theoretical constructions, whose relevance to nature is still a big question mark, QCD will stay with us forever. I am happy that I invested so much time and effort in studying QCD. This was a long and exciting journey. Almost 40 years later, I am honored and proud to be invited to this Conference celebrating Professor Gell-Mann's 80 ${ }^{\text {th }}$ birthday to give a talk on advances in QCD.

I should say that the problem of strong interactions turned out to be extremely difficult (despite the fact that the underlying Lagrangian is firmly established) and the advances slow and painful. This is a usual story with the strong coupling regime: whenever theorists find themselves at strong coupling, they are in trouble. YangMills theories are no exception.

My task today is to outline some contours of the strong interaction theory which gradually emerged from obscurity during these four decades. Yes, the theory is

\footnotetext{
${ }^{1}$ Institute for Theoretical and Experimental Physics in Moscow
} 


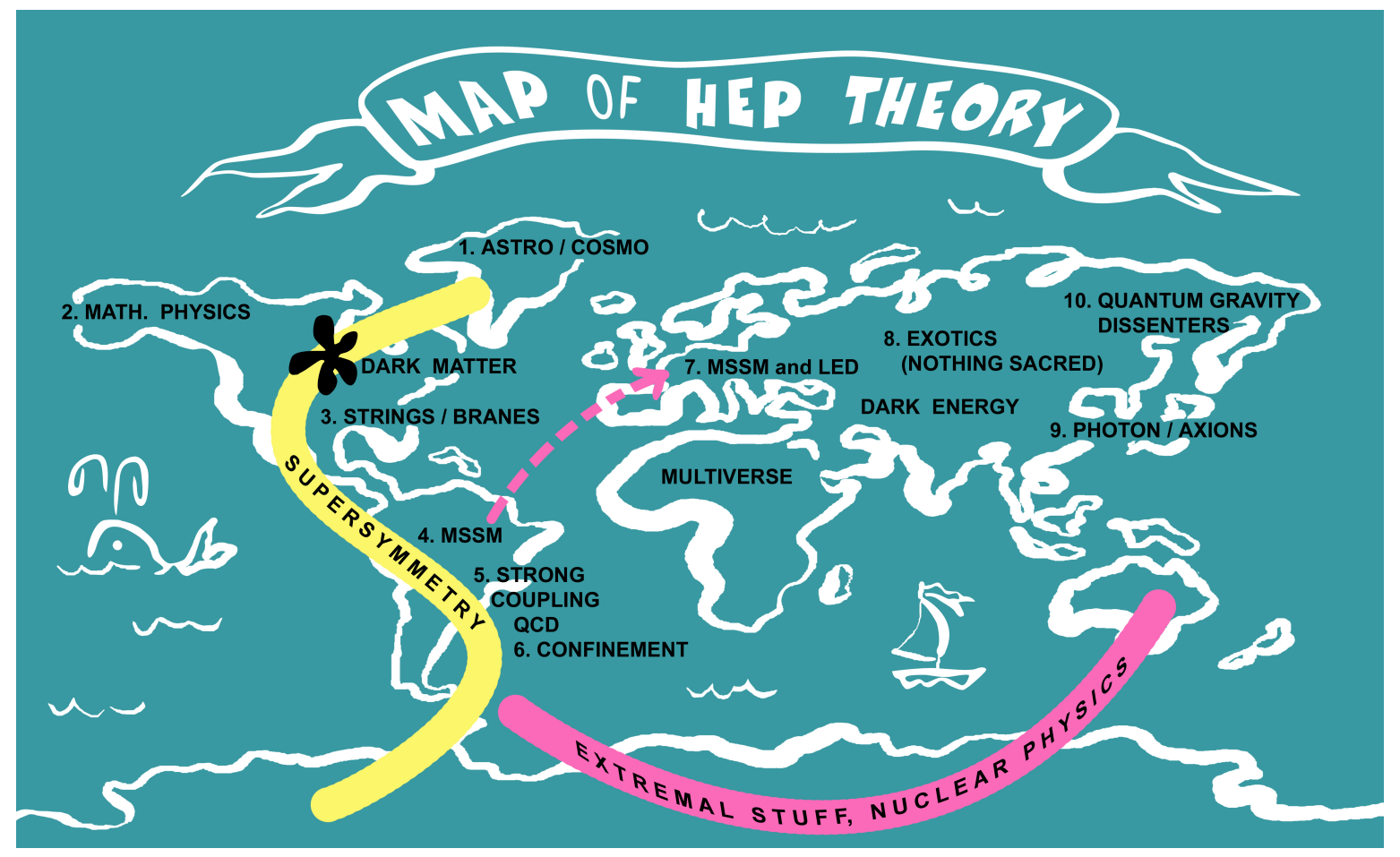

Figure 2: Map of the HEP theory.

incomplete, but those parts which are already in existence are beautiful, and continue to grow.

First, to give a general idea of the role which this theory plays in high energy physics (HEP) I would like to chart approximate contours of main areas that are under intense development in the theoretical community of today. To this end I display a symbolic map in Fig. 2. The linkage of the HEP theory map to the earth's geography is arbitrary and does not mean anything. You should pay attention only to interconnections of various areas of HEP. You see that QCD and strongly coupled gauge theories at large, in which Murray Gell-Mann was a trailblazer, occupy a vast area. The advances there and in neighboring areas crucially depend on the exchange of ideas between them. One can say that they feed each other. Of particular importance was a breakthrough impact of supersymmetry of which I will speak later. In the Appendix you can find some additional information regarding the HEP theory map obtained from a HEP world traveller. Unfortunately, the traveller was unable to visit some areas (allegedly, because of clearance issues), namely, that of extremal 
phenomena (high-energy and high density QCD), nuclear physics, multiverses, and theoretical nonperturbative supersymmtry.

\section{QCD}

In all processes with hadron participation strong interactions play a role. All matter surrounding us is made of protons and neutrons the most common representatives of the class of hadrons. Even if you do not see them explicitly, they will necessarily show up at a certain stage or in loops. The fundamental Lagrangian governing strong interactions is

$$
\mathcal{L}=\sum_{f} \bar{\psi}_{f}\left(i \not D-m_{f}\right) \psi^{f}-\frac{1}{4} G_{\mu \nu}^{a} G^{\mu \nu a}
$$

The first term describes color-triplet quarks and their coupling to color-octet gluons. It ascends to Gell-Mann. The second Yang-Mills term describes the gluon dynamics. Both terms taken together comprise the Lagrangian of quantum chromodynamics (QCD). The very name "quantum chromodynamics" ascends to Gell-Mann too. Much in the same way as the Schrödinger equation codes all of quantum chemistry, the QCD Lagrangian codes all of

- nuclear physics;

- Regge behavior;

- neutron stars;

- chiral physics;

- light \& heavy quarkonia;

- glueballs \& exotics;

- exclusive \& inclusive hadronic scattering at large momentum transfer;

- interplay between strong forces \& weak interactions,

- quark-gluon plasma;

and much more.

Although the underlying Lagrangian (1), and asymptotic freedom it implies at short distances [1], are established beyond any doubt, the road from this starting point to theoretical control over the large-distance hadronic world is long and difficult. The journey which started 40 years ago is not yet completed. En route, many beautiful theoretical constructions were developed allowing one to understand various corners of the hadronic world. Here I am unable even to list them, let alone discuss in 
a comprehensible way. Therefore, I will focus only on one - albeit absolutely global

- aspect defining the hadronic world: the confinement phenomenon.

\section{Confinement in Non-Abelian Gauge Theories: dual Meissner Effect}

The most salient feature of pure Yang-Mills theory is linear confinement. If one takes a heavy probe quark and an antiquark separated by a large distance, the force between them does not fall off with distance, while the potential energy grows linearly. This is the explanation of the empiric fact that quarks and gluons (the microscopic degrees of freedom in QCD) never appear as asymptotic states. The physically observed spectrum consists of color-singlet mesons and baryons. The phenomenon got the name color confinement, or, in a more narrow sense, quark confinement. In the early days of QCD it was also referred to as infrared slavery.

Quantum chromodynamics (QCD), and Yang-Mills theories at strong coupling at large, are not yet analytically solved. Therefore, it is reasonable to ask:

Are there physical phenomena in which interaction energy between two interacting bodies grows with distance at large distances? Do we understand the underlying mechanism?

The answer to these questions is positive. The phenomenon of linearly growing potential was predicted by Abrikosov [2] in the superconductors of the second type. The corresponding set up is shown in Fig. 3. In the middle of this figure we see a superconducting sample, with two very long magnets attached to it. The superconducting medium does not tolerate the magnetic field. On the other hand, the flux of the magnetic field must be conserved. Therefore, the magnetic field lines emanating from the $N$ pole of one magnet find their way to the $S$ pole of another magnet, through the medium, by virtue of a flux tube formation. Inside the flux tube the Cooper pair condensate vanishes and superconductivity is destroyed. The flux tube has a fixed tension, implying a constant force between the magnetic poles as long as they are inside the superconducting sample. The phenomenon described above is sometimes referred to as the Meissner effect.

Of course, the Meissner effect of the Abrikosov type occurs in the Abelian theory, QED. The flux tube that forms in this case is Abelian. In Yang-Mills theories we are interested in non-Abelian analogs of the Abrikosov vortices. Moreover, while in 


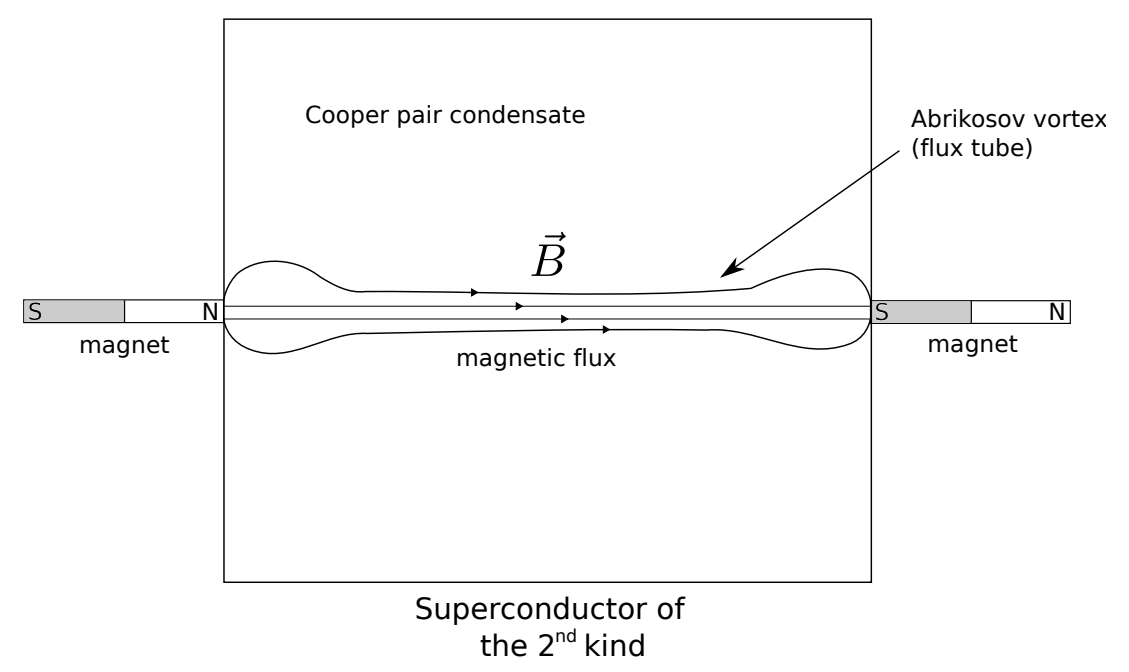

Figure 3: The Meissner effect in QED.

the Abrikosov case the flux tube is that of the magnetic field, in QCD and QCD-like theories the confined objects are quarks; therefore, the flux tubes must be "chromoelectric" rather than chromomagnetic. In the mid-1970s Nambu, 't Hooft, and Mandelstam (independently) put forward an idea [3] of a "dual Meissner effect" as the underlying mechanism for color confinement. Within their conjecture, in chromoelectric theories "monopoles" condense leading to formation of "non-Abelian flux tubes" between the probe quarks. At this time the Nambu-'t Hooft-Mandelstam paradigm was not even a physical scenario, rather a dream, since people had no clue as to the main building blocks such as non-Abelian flux tubes. After the Nambu-'t Hooft-Mandelstam conjecture had been formulated many works were published on this subject, to no avail.

A long-awaited breakthrough discovery came 20 years later: the Seiberg-Witten solution [4] of $\mathcal{N}=2$ super-Yang-Mills theory slightly deformed by a superpotential breaking $\mathcal{N}=2$ down to $\mathcal{N}=1$. In the $\mathcal{N}=2$ limit, the theory has a moduli space. If the gauge group is $\mathrm{SU}(2)$, on the moduli space, $\mathrm{SU}(2)_{\text {gauge }}$ is spontaneously broken down to U(1). Therefore, the theory possesses the 't Hooft-Polyakov monopoles [5] in the quasiclassical regime. Of course, in this regime they are very heavy and play no role in dynamics. Using the power of $\mathcal{N}=2$ supersymmetry, two special points on the moduli space were found [4] at strong coupling, (the monopole and dyon points), in which the monopoles (dyons) become massless. In these points the scale of the 
gauge symmetry breaking

$$
\mathrm{SU}(2) \rightarrow \mathrm{U}(1)
$$

is determined by $\Lambda$, the dynamical scale parameter of $\mathcal{N}=2$ super-Yang-Mills theory.

All physical states can be classified with regards to the unbroken $\mathrm{U}(1)$. It is natural to refer to the $\mathrm{U}(1)$ gauge boson as to the photon. In addition to the photon, all its superpartners, being neutral, remain massless at this stage, while all other states, with nonvanishing "electric" charges, acquire masses of the order of $\Lambda$. In particular, two gauge bosons corresponding to $\mathrm{SU}(2) / \mathrm{U}(1)$ - it is natural to call them $W^{ \pm}$- have masses $\sim \Lambda$. All such states are "heavy" and can be integrated out.

In the low-energy limit, near the monopole and dyon points, one deals with electrodynamics of massless monopoles. One can formulate an effective local theory describing interaction of the light states by dualizing the original phton. This is a $\mathrm{U}(1)$ gauge theory in which the (magnetically) charged matter fields $M, \tilde{M}$ are those of monopoles while the $\mathrm{U}(1)$ gauge field that couples to $M, \tilde{M}$ is dual with respect to the photon of the original theory. The $\mathcal{N}=2$ preserving superpotential has the form $\mathcal{W}=\mathcal{A} M \tilde{M}$, where $\mathcal{A}$ is the $\mathcal{N}=2$ superpartner of the dual photon/photino fields.

Now, if one switches on a small $\mathcal{N}=2$ breaking superpotential, the only change in the low-energy theory is the emergence of the extra $m^{2} \mathcal{A}$ term in the superpotential. Its impact is crucial: it triggers the monopole condensation, $\langle M\rangle=\langle\tilde{M}\rangle=m$, which implies, in turn, that the dual U(1) symmetry is spontaneously broken, and the dual photon acquires a mass $\sim m$. As a consequence, the Abrikosov flux tubes are formed. Viewed inside the dual theory, they carry fluxes of the magnetic field. With regards to the original microscopic theory these are the electric field fluxes.

Thus, Seiberg and Witten demonstrated, for the first time ever, the existence of the dual Meissner effect in a judiciously chosen non-Abelian gauge field theory. If one "injects" a probe (very heavy) quark and antiquark in this theory, a flux tube forms between them, with necessity, leading to linear confinement.

The flux tubes in the Seiberg-Witten solution were investigated in detail in [6]. These flux tubes are Abelian, and so is confinement caused by their formation. What does that mean? At the scale of distances at which the flux tube is formed (the inverse mass of the Higgsed U(1) photon) the gauge group that is operative is Abelian. In 
the Seiberg-Witten analysis this is the dual U(1). The off-diagonal (charged) gauge bosons are very heavy in this scale and play no direct role in the flux tube formation and confinement that ensues. Naturally, the spectrum of composite objects in this case turns out to be richer than that in QCD and similar theories with non-Abelian confinement. By non-Abelian confinement I mean such dynamical regime in which at distances of the flux tube formation all gauge bosons are equally important.

Moreover, the string topological stability is based on $\pi_{1}(\mathrm{U}(1))=\mathbb{Z}$. Therefore, $N$ strings do not annihilate as they should in $\mathrm{SU}(N)$ QCD-like theories.

The two-stage symmetry breaking pattern, with $\mathrm{SU}(2) \rightarrow \mathrm{U}(1)$ occurring at a high scale while the dual $\mathrm{U}(1) \rightarrow$ nothing at a much lower scale, has no place in QCD-like theories, as we know from experiment. In such theories, presumably, all non-Abelian gauge degrees of freedom take part in the string formation, and are operative at the scale at which the strings are formed. The strings in the SeibergWitten solution are believed to belong to the same universality class as those in QCD-like theories. However, in the limit of large- $m$ deformations, when a nonAbelian regime presumably sets in and non-Abelian strings develop in the model considered by Seiberg and Witten, theoretical control is completely lost. Thus, the status of the statement of the same universality class is conjectural.

\section{Non-Abelian strings}

In a bid to better understand string-based confinement mechanism in Yang-Mills theories that might be more closely related to QCD people continued searches for models supporting non-Abelian strings. If a model in which non-Abelian strings develop in a fully controllable manner, i.e. at weak coupling, could be found and the passage from Abelian to non-Abelian strings explored, this would provide us with evidence that no phase transition occurs between the two regimes in the SeibergWitten solution.

In the technical sense, what does one mean when one speaks of non-Abelian flux tubes? Apparently, the orientation of the magnetic field in the tube interior must be free to strongly fluctuate inside the $\mathrm{SU}(N)$ group. There is no such freedom in the Abelian string of the Abrikosov type. In other words, in addition to translational moduli, the theory on the string world sheet must acquire orientational moduli (Fig. 4). 


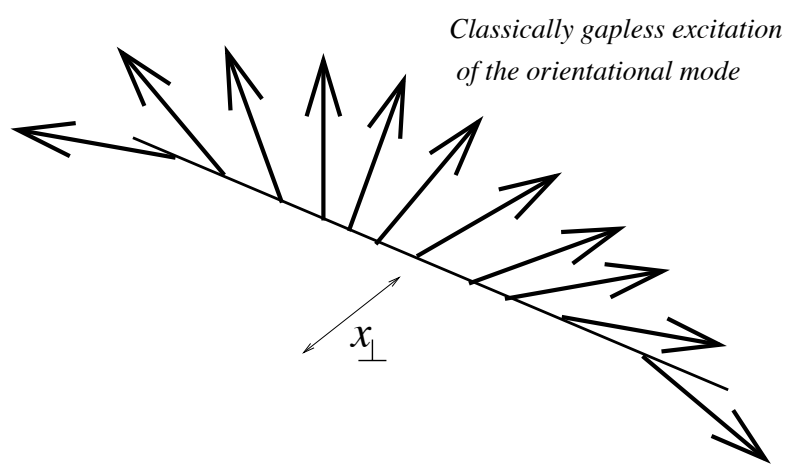

Figure 4: Orientational moduli on the string world sheet.

If one thinks there is a kind of string theory behind QCD confining dynamics, such behavior is natural. Indeed, string theory is formulated in higher dimensions. Bringing it to $D=4$ requires compactification of some dimensions. If (some of) compact dimensions have isometries, the corresponding sigma model on the 4D string world sheet will have classically massless internal degrees of freedom. For instance, compactification on $S_{2}$ gives rise to $\mathrm{CP}(1)$ sigma model on the string world sheet. Two-dimensional infrared dynamics will then generate a mass gap for these orientational degrees of freedom.

That's why searches for non-Abelian flux tubes and non-Abelian monopoles in the bulk Yang-Mills theories continued, with a decisive breakthrough in 2003-04 [7, 8. By that time the program of finding field-theoretical analogs of all basic constructions of string/D-brane theory was in full swing. BPS domain walls, analogs of D branes, had been identified in supersymmetric Yang-Mills theory [9]. It had been demonstrated that such walls support gauge fields localized on them. BPS saturated string-wall junctions had been constructed [10]. Topological stability of the non-Abelian strings under consideration is due to the fact that

$$
\pi_{1}\left(\frac{\mathrm{SU}(2) \times \mathrm{U}(1)}{Z_{2}}\right) \rightarrow \text { nontrivial }
$$

\section{Basic Bulk Theory: setting the stage}

Non-Abelian strings were first found in $\mathcal{N}=2$ super-Yang-Mills theories with $\mathrm{U}(2)_{\text {gauge }}$ and two matter hypermultiplets [7, 8]. The $\mathcal{N}=2$ vector multiplet consists 
of the $\mathrm{U}(1)$ gauge field $A_{\mu}$ and the $\mathrm{SU}(2)$ gauge field $A_{\mu}^{a}$, (here $a=1,2,3$ ), and their Weyl fermion superpartners $\left(\lambda^{1}, \lambda^{2}\right)$ and $\left(\lambda^{1 a}, \lambda^{2 a}\right)$, plus complex scalar fields $a$, and $a^{a}$. The global $\mathrm{SU}(2)_{R}$ symmetry inherent to $\mathcal{N}=2$ models manifests itself through rotations $\lambda^{1} \leftrightarrow \lambda^{2}$.

The quark multiplets consist of the complex scalar fields $q^{k A}$ and $\tilde{q}_{A k}$ (squarks) and the Weyl fermions $\psi^{k A}$ and $\tilde{\psi}_{A k}$, all in the fundamental representation of the $\mathrm{SU}(2)$ gauge group $(k=1,2$ is the color index while $A$ is the flavor index, $A=1,2)$. The scalars $q^{k A}$ and $\overline{\tilde{q}}^{k A}$ form a doublet under the action of the global SU(2) $R$ group. The quarks and squarks have a U(1) charge too.

If one introduces a non-vanishing Fayet-Iliopoulos parameter $\xi$ the theory develops isolated quark vacua, in which the gauge symmetry is fully Higgsed, and all elementary excitations are massive. In the general case, two matter mass terms allowed by $\mathcal{N}=2$ are unequal, $m_{1} \neq m_{2}$. There are free parameters whose interplay determines dynamics of the theory: the Fayet-Iliopoulos parameter $\xi$, the mass difference $\Delta m$ and a dynamical scale parameter $\Lambda$, an analog of the QCD scale $\Lambda_{\mathrm{QCD}}$ (Fig. 5). Extended supersymmetry guarantees that some crucial dependences are holomorphic, and there is no phase transition.

Both the gauge and flavor symmetries of the model are broken by the squark condensation. All gauge bosons acquire the same masses (which are of the order of inverse string thickness). A global diagonal combination of color and flavor groups, $\mathrm{SU}(2)_{C+F}$, survives the breaking (the subscript $C+F$ means a combination of global color and flavor groups).

While $\mathrm{SU}(2)_{C+F}$ is the symmetry of the vacuum, the flux tube solutions break it spontaneously. This gives rise to orientational moduli on the string world sheet.

The bulk theory is characterized by three parameters of dimension of mass: $\xi$, $\Delta m$, and $\Lambda$. As various parameters vary, the theory under consideration evolves in a very graphic way, see Fig. 5. At $\xi=0$ but $\Delta m \neq 0$ (and $\Delta m \gg \Lambda$ ) it presents a very clear-cut example of a model with the standard 't Hooft-Polyakov monopole. This is due to the fact that the relevant part of the bosonic sector is nothing but the Georgi-Glashow model. The monopole is unconfined - the flux tubes are not yet formed.

Switching on $\xi \neq 0$ traps the magnetic fields inside the flux tubes, which are weak as long as $\xi \ll \Delta m$. The flux tubes change the shape of the monopole far away from its core, leaving the core essentially intact. Orientation of the chromomagnetic field inside the flux tube is essentially fixed. This is due to the fact that all off-diagonal 


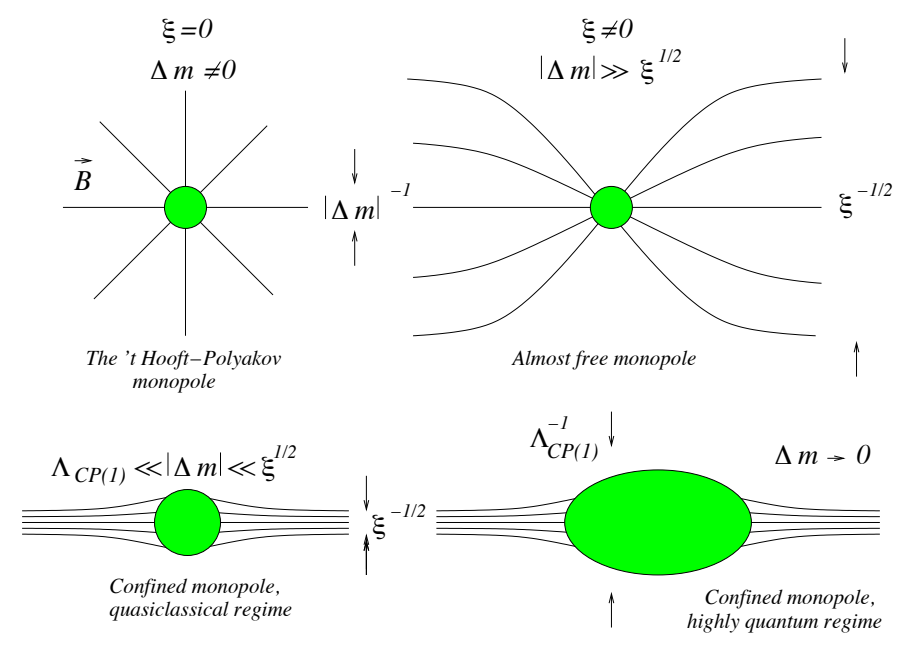

Figure 5: Various regimes for monopoles and strings.

gauge bosons ( $W$ bosons) are heavy in this limit. Thus, the flux tubes supported in this limit are Abelian. (They are commonly referred to as the $Z_{N}$ strings.)

With $|\Delta m|$ decreasing, fluctuations in the orientation of the chromomagnetic field inside the flux tubes grow. Simultaneously, the monopole which no loner resembles the 't Hooft-Polyakov monopole, is seen as a string junction.

Finally, in the limit $\Delta m \rightarrow 0$ the transformation is complete. A global SU(2) symmetry restores in the bulk. All three gauge bosons have identical masses. Orientational (exact, classically massless) moduli develop on the string world sheet making it non-Abelian. The string world sheet theory is $\mathrm{CP}(1)(\mathrm{CP}(N-1)$ for generic values of $N)$. Two-dimensional $\mathrm{CP}(N-1)$ models with four supercharges are asymptotically free. They have $N$ distinct vacuum states.

Each vacuum state of the worldsheet $\mathrm{CP}(N-1)$ theory presents a distinct string from the standpoint of the bulk theory. There are $N$ species of such strings; they have degenerate tensions $T_{\mathrm{st}}=2 \pi \xi$. The ANO string tension is $N$ times larger.

Two different strings can form a stable junction. Figure 6 shows this junction in the limit

$$
\Lambda_{\mathrm{CP}(1)} \ll|\Delta m| \ll \sqrt{\xi}
$$

corresponding to the lower left corner in Fig. 5. The magnetic fluxes of the U(1) and $\mathrm{SU}(2)$ gauge groups are oriented along the $z$ axis. In the limit (4) the $\mathrm{SU}(2)$ flux 


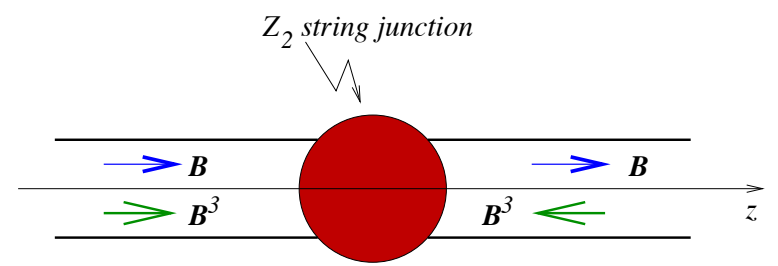

Figure 6: $Z_{2}$ string junction.

is oriented along the third axis in the internal space. However, as $|\Delta m|$ decreases, fluctuations of $B_{z}^{a}$ in the internal space grow, and at $\Delta m \rightarrow 0$ it has no particular orientation in $\mathrm{SU}(2)$ (the lower right corner of Fig. 5). In the language of the worldsheet theory this phenomenon is due to restoration of the $\mathrm{O}(3)$ symmetry in the quantum vacuum of the $\mathrm{CP}(1)$ model.

Evolution from the upper right corner in Fig. 5 to the lower right corner is in fact the transformation of the Abelian string into non-Abelian. $\mathcal{N}=2$ supersymmetry guarantees that it is smooth, with no phase transition.

The junctions of degenerate strings present what remains of the monopoles in this highly quantum regime [11, 12]. It is remarkable that, despite the fact we are deep inside the highly quantum regime, holomorphy allows one to exactly calculate the mass of these monopoles. This mass is given by the expectation value of the kink central charge in the worldsheet $\mathrm{CP}(N-1)$ model (including the anomaly term), $M_{M} \sim N^{-1}\left\langle R \psi_{L}^{\dagger} \psi_{R}\right\rangle$.

\section{Towards $\mathcal{N}=1$}

The "unwanted" feature of $\mathcal{N}=2$ theory, making it less similar to QCD than one would desire, is the presence of the adjoint chiral superfields $\mathcal{A}$ and $\mathcal{A}^{a}$. One can get rid of them making them heavy. To this end we can endow the adjoint superfield with a mass term of the type $\mu \mathcal{A}^{2}$, through the $\mathcal{N}=1$ preserving superpotential

$$
\mathcal{W}=\frac{\mu}{2}\left[\mathcal{A}^{2}+\left(\mathcal{A}^{a}\right)^{2}\right] .
$$

Now, supersymmetry of the bulk model becomes $\mathcal{N}=1$. At large $\mu$ the adjoint fields decouple. 
With the deformation superpotential (5) the 1/2 BPS classical flux tube solution stays the same as in the absence of this superpotential [13]. Moreover, the number of the boson and fermion zero modes, which become moduli fields on the string world sheet, does not change either. For the fermion zero modes this statement follows from an index theorem proved in [14]. If the string solution and the number of zero modes remain the same, what can one say about the string world-sheet theory?

The bulk deformation (5) leads to a remarkable, heterotic deformation of the $\mathrm{CP}(1)$ model on the world sheet, with $\mathcal{N}=(0,2)$ supersymmetry. The discovery of non-Abelian strings in $\mathcal{N}=1$ bulk theories is a crucial step on the way to the desired $\mathcal{N}=0$ theories. Moreover, the heterotically deformed $\mathrm{CP}(1)$ model is very rich by itself exhibiting a number of distinct dynamical scenarios unknown previously.

To understand the emergence of $\mathcal{N}=(0,2)$ supersymmetry in the world-sheet Lagrangian recall that $\mathcal{N}=2$ Yang-Mills theories which support non-Abelian flux tubes have eight supercharges. The flux tube solutions are 1/2 BPS-saturated. Hence, the effective low-energy theory of the moduli fields on the string world sheet must have four supercharges. The bosonic moduli consist of two groups: two translational moduli $\left(x_{0}\right)_{1,2}$ corresponding to translations in the plane perpendicular to the string axis, and two orientational moduli whose interaction is described by $\mathrm{CP}(1)$ (see Fig. (4). The fermion moduli also split in two groups: four supertranslational moduli $\zeta_{L}, \zeta_{L}^{\dagger}, \zeta_{R}, \zeta_{R}^{\dagger}$ plus four superorientational moduli. $\mathcal{N}=2$ supersymmetry in the bulk and on the worldsheet guarantees that $\left(x_{0}\right)_{1,2}$ and $\zeta_{L, R}$ form a free field theory on the worldsheet completely decoupling from (super)orientational moduli, which in turn form $\mathcal{N}=(2,2)$ supersymmetric $\mathrm{CP}(1)$ model.

When one deforms the bulk theory to break $\mathcal{N}=2$ down to $\mathcal{N}=1$, one has four supercharges in the bulk and expects two supercharges on the world sheet. Two out of four supertranslational modes, $\zeta_{R}$ and $\zeta_{R}^{\dagger}$, get coupled to two superorientational modes $\psi_{R}$ and $\psi_{R}^{\dagger}$ [16]. At the same time, $\zeta_{L}$ and $\zeta_{L}^{\dagger}$ remain protected. Thus, the right- and left-moving fermions acquire different interactions; hence, the flux tube becomes heterotic!

This breaks two out of four supercharges on the world sheet. Edalati and Tong outlined [16] a general structure of the chiral $\mathcal{N}=(0,2)$ generalization of $\mathrm{CP}(1)$. Derivation of the heterotic $\mathrm{CP}(1)$ model from the bulk theory was carried out in Ref. [15]. 


\section{$7 \quad$ Heterotic non-Abelian string}

The Lagrangian of the heterotic $\mathrm{CP}(N-1)$ model can be written as [15]

$$
\begin{aligned}
L_{\text {heterotic }} & =\zeta_{R}^{\dagger} i \partial_{L} \zeta_{R}+\left[\gamma g_{0}^{2} \zeta_{R} G_{i \bar{j}}\left(i \partial_{L} \phi^{\dagger \bar{j}}\right) \psi_{R}^{i}+\text { H.c. }\right] \\
& -g_{0}^{4}|\gamma|^{2}\left(\zeta_{R}^{\dagger} \zeta_{R}\right)\left(G_{i \bar{j}} \psi_{L}^{\dagger \bar{j}} \psi_{L}^{i}\right) \\
& +G_{i \bar{j}}\left[\partial_{\mu} \phi^{\dagger \bar{j}} \partial_{\mu} \phi^{i}+i \bar{\psi}^{\bar{j}} \gamma^{\mu} D_{\mu} \psi^{i}\right] \\
& -\frac{g_{0}^{2}}{2}\left(G_{i \bar{j}} \psi_{R}^{\dagger \bar{j}} \psi_{R}^{i}\right)\left(G_{k \bar{m}} \psi_{L}^{\dagger \bar{m}} \psi_{L}^{k}\right) \\
& +\frac{g_{0}^{2}}{2}\left(1-2 g_{0}^{2}|\gamma|^{2}\right)\left(G_{i \bar{j}} \psi_{R}^{\dagger \bar{j}} \psi_{L}^{i}\right)\left(G_{k \bar{m}} \psi_{L}^{\dagger \bar{m}} \psi_{R}^{k}\right) .
\end{aligned}
$$

The constant $\gamma$ in Eq. (6) is the parameter which determines the "strength" of the heterotic deformation, and the left-right asymmetry in the fermion sector. It is related to the parameter $\mu$ in Eq. (5) (e.g. $\gamma \propto \mu$ at small $\mu$ ). The third, fourth and fifth lines in Eq. (6) are the same as in the conventional $\mathcal{N}=(2,2) \operatorname{CP}(N-1)$ model, except the last coefficient.

Introduction of a seemingly rather insignificant heterotic deformation drastically changes dynamics of the $\mathrm{CP}(1)$ model, leading to spontaneous supersymmetry breaking. At small $\mu$ (small $\gamma)$ the field $\zeta_{R}$ represents a massless Goldstino, with the residue $\left\langle R \psi_{R}^{\dagger} \psi_{L}\right\rangle$. As well known, a nonvanishing bifermion condensate $\left\langle R \psi_{R}^{\dagger} \psi_{L}\right\rangle$ develops in the undeformed model. Thus, the vacuum energy

$$
\mathcal{E}_{\mathrm{vac}}=|\gamma|^{2}\left|\left\langle R \psi_{R}^{\dagger} \psi_{L}\right\rangle\right|^{2} \neq 0
$$

Therefore, upersymmetry is spontaneously broken. A nonvanishing $\mathcal{E}_{\mathrm{vac}}$ for arbitrary values of $\gamma$ in heterotically deformed $\mathrm{CP}(N-1)$ models was obtained in [17] from the large- $N$ expansion. Spontaneous breaking of SUSY in heterotic $C P(N-1)$ was anticipated in [18]. 


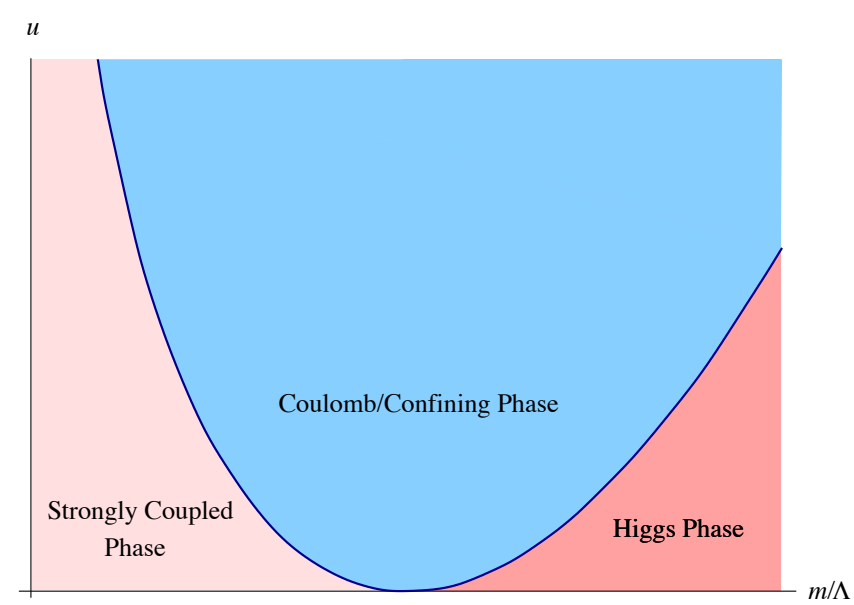

Figure 7: The phase diagram of the twisted-mass deformed heterotic $\operatorname{CP}(N-1)$ theory. The parameter $u$ denotes the amount of deformation and is related to $\gamma$.

\section{Large- $N$ solution of the heterotic $\mathbf{C P}(N-1)$ model}

To reveal a rich dynamical structure of the heterotically deformed $\mathrm{CP}(N-1)$ models it is instructive to add twisted masses which correspond to $\Delta m \neq 0$ introduced above. Moreover, the most convenient choice of the twisted masses is that preserving the $Z_{N}$ symmetry of the model which exists at $\Delta m=0$,

$$
m_{k}=m \exp \left(i \frac{2 \pi k}{N}\right), \quad k=0,1,2, \ldots, N-1 .
$$

where $m$ is a complex parameter setting the scale of the twisted masses. For simplicity I will take it real. Now we have two variable parameters, $m$ and $\gamma$, the strength of the heterotic deformation. The breaking vs. nonbreaking of the above $Z_{N}$ determines the phase diagram. This model can be solved at large $N$ using the $1 / N$ expansion [17]. I will present here just two plots exhibiting main features of the solution.

Figure 7 displays three distinct regimes and two phase transition lines. Two phases with the spontaneously broken $Z_{N}$ on the left and on the right are separated by a phase with unbroken $Z_{N}$. This latter phase is characterized by a unique vacuum and confinement of all U(1) charged fields ("quarks"). In the broken phases (one of them is at strong coupling) there are $N$ degenerate vacua and no confinement.

Figure 8 shows the vacuum energy density at a fixed value of $\gamma$. It demonstrates that supersymmetry is spontaneously broken everywhere except a circle $|m|=\Lambda$ in 


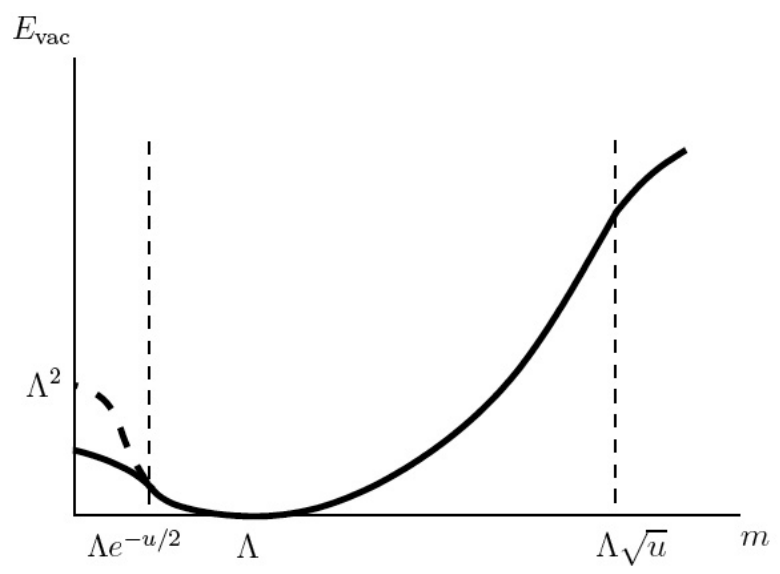

Figure 8: Vacuum energy density vs. $m$. The dashed line shows an unstable quasivacuum.

the $Z_{N}$-unbroken phase. The first phase transition occurs at strong coupling (small $|m|$ ) while the second phase transition is at weak coupling (large $|m|$ ). Both phase transitions between the three distinct phases are of the second kind.

One must be able to translate this rich world sheet-dynamics into (presumably) highly nontrivial statements regarding the bulk theory at strong coupling.

\section{Instead of conclusion}

The progress in understanding dynamics of non-Abelian theories at strong coupling was painfully slow. But what a progress it is! To properly appreciate the scale of achievements, please, look back in the 1970's and compare what was known then about strong interactions to what we know now. Just open old reviews or textbooks devoted to this subject, in parallel with fresh publications. Of course, a pessimist might say that the full analytical theory is still elusive. Will it ever be created? And what does it mean, "the full analytical theory," in the case when we are at strong coupling? The richness of the hadronic world is enormous. Unlike QED we will never be able to analytically calculate all physical observables with arbitrary precision. But do we really need this? To my mind, what is really needed is the completion of the overall qualitative picture of confinement in non-supersymmetric theories, supplemented by a variety of approximate quantitative tools custom-designed to treat particular applications. A large number of such tools are already available. 


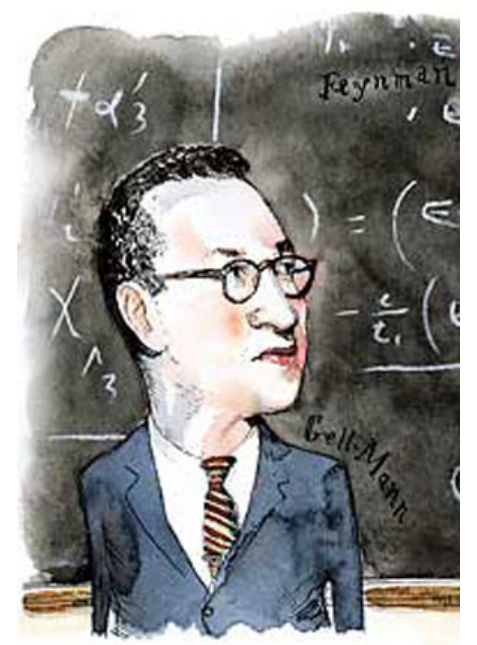

Figure 9: Everybody knows that this is Murray Gell-Mann. (C)Barry Blitt, The Atlantic Monthly, July 2000.)

\section{Acknowledgments}

I am very grateful to A. Yung with whom I shared the pleasure of working on the issues discussed in this talk. Generous assistance of Andrey Feldshteyn with cartoons is gratefully acknowledged. This write-up was completed during my stay at the Institut de Physique Théorique, CEA-Saclay, which was made possible due to the support of the Chaires Internacionales de Recherche Blaise Pascal, Fondation de l'Ecole Normale Supérieure. This work is supported in part by the DOE grant DE-FG02-94ER408.

\section{References}

[1] D. J. Gross and F. Wilczek, Phys. Rev. Lett. 30, 1343 (1973); H. D. Politzer, Phys. Rev. Lett. 30, 1346 (1973).

[2] A. Abrikosov, Sov. Phys. JETP 32, 1442 (1957) [Reprinted in Solitons and Particles, Eds. C. Rebbi and G. Soliani (World Scientific, Singapore, 1984), p. 356];

H. Nielsen and P. Olesen, Nucl. Phys. B61, 45 (1973) [Reprinted in Solitons and Particles, Eds. C. Rebbi and G. Soliani (World Scientific, Singapore, 1984), p. 365]. 
[3] Y. Nambu, Phys. Rev. D 10, 4262 (1974);

G. 't Hooft, Gauge theories with unified weak, electromagnetic and strong interactions, in Proc. of the E.P.S. Int. Conf. on High Energy Physics, Palermo, 23-28 June, 1975 ed. A. Zichichi (Editrice Compositori, Bologna, 1976); S. Mandelstam, Phys. Rept. 23, 245 (1976).

[4] N. Seiberg and E. Witten, Nucl. Phys. B426, 19 (1994), (E) B430, 485 (1994) hepth/9407087]; Nucl. Phys. B431, 484 (1994) [hep-th/9408099].

[5] G. 't Hooft, Nucl. Phys. B 79, 276 (1974);

A. M. Polyakov, JETP Lett. 20, 194 (1974).

[6] M. R. Douglas and S. H. Shenker, Nucl. Phys. B 447, 271 (1995) arXiv:hepth/9503163; A. Hanany, M. J. Strassler and A. Zaffaroni, Nucl. Phys. B 513, 87 (1998) hep-th/9707244.

[7] A. Hanany and D. Tong, JHEP 0307, 037 (2003) hep-th/0306150.

[8] R. Auzzi, S. Bolognesi, J. Evslin, K. Konishi and A. Yung, Nucl. Phys. B 673, 187 (2003) hep-th/0307287.

[9] G. R. Dvali and M. A. Shifman, Phys. Lett. B 396, 64 (1997), (E) B 407, 452 (1997) arXiv:hep-th/9612128.

[10] M. Shifman and A. Yung, Phys. Rev. D 67, 125007 (2003) arXiv:hep-th/0212293.

[11] M. Shifman and A. Yung, Phys. Rev. D 70, 045004 (2004) hep-th/0403149.

[12] A. Hanany and D. Tong, JHEP 0404, 066 (2004) hep-th/0403158.

[13] M. Shifman and A. Yung, Phys. Rev. D 72, 085017 (2005) arXiv:hep-th/0501211.

[14] A. Gorsky, M. Shifman and A. Yung, Phys. Rev. D 75, 065032 (2007) arXiv:hepth/0701040.

[15] M. Shifman and A. Yung, Phys. Rev. D 77, 125016 (2008) [Erratum D 79, 049901 (2009)] arXiv:0803.0158 [hep-th]].

[16] M. Edalati and D. Tong, JHEP 0705, 005 (2007) arXiv:hep-th/0703045.

[17] M. Shifman and A. Yung, Large- $N$ Solution of the Heterotic $\mathcal{N}=(0,2)$ TwoDimensional CP(N-1) Model, arXiv:0803.0698 [hep-th].

[18] D. Tong, JHEP 0709, 022 (2007) arXiv:hep-th/0703235. 


\section{Appendix: Snapshots made by HEP world traveller}

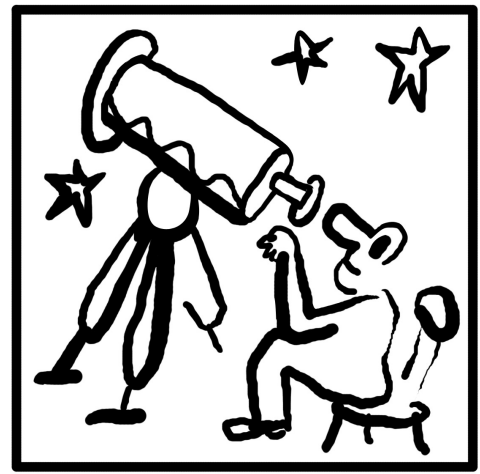

1. ASTRO / COSMO

Figure 10: Astroparticle physics and cosmology.

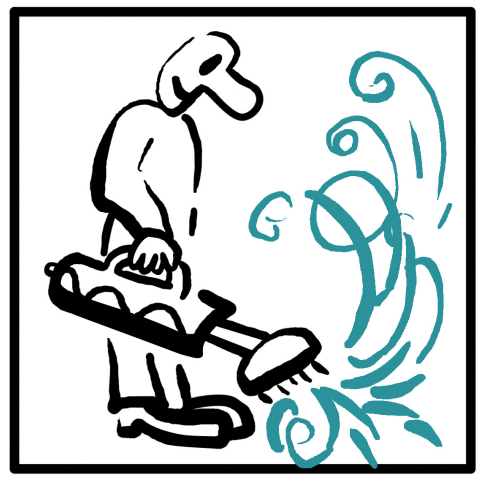

2. MATH. PHYSICS

Figure 11: Mathematical physics absorbing string theory. 


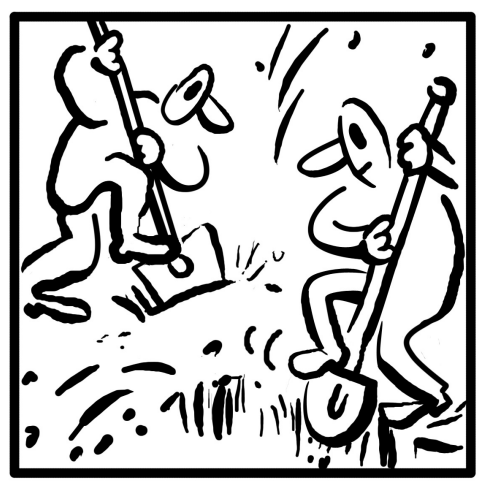

3. STRINGS / BRANES

Figure 12: The theory of strings and branes.

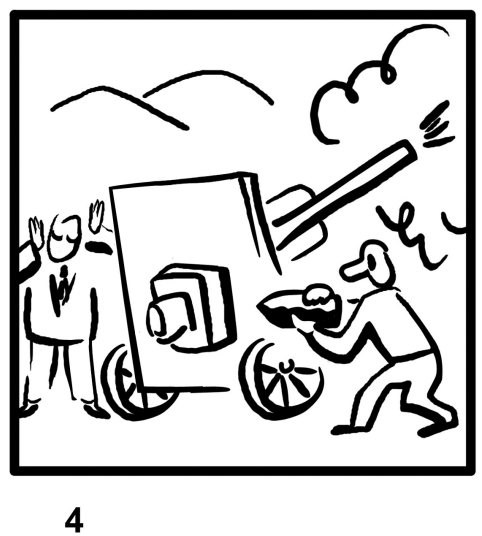

Figure 13: Supersymmetry-based phenomenology. 


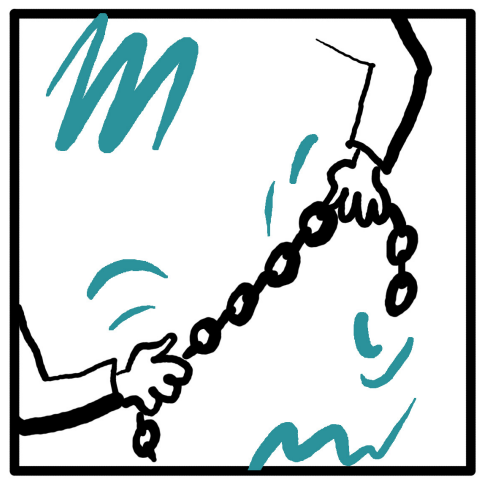

5. STRONG COUPLING

Figure 14: QCD and other gauge theories at strong coupling: from confinement to chiral symmetry breaking and back via supersymmetry.

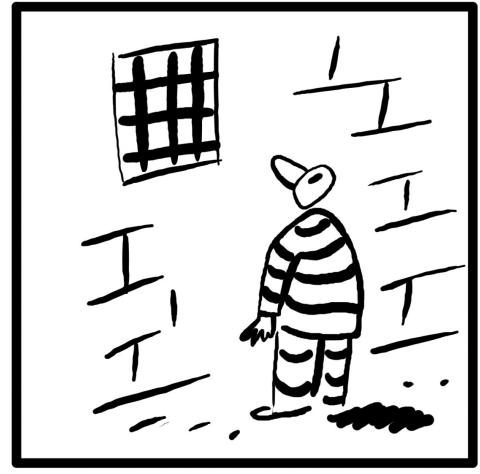

6. CONFINEMENT

Figure 15: Deciphering mechanisms of confinement 


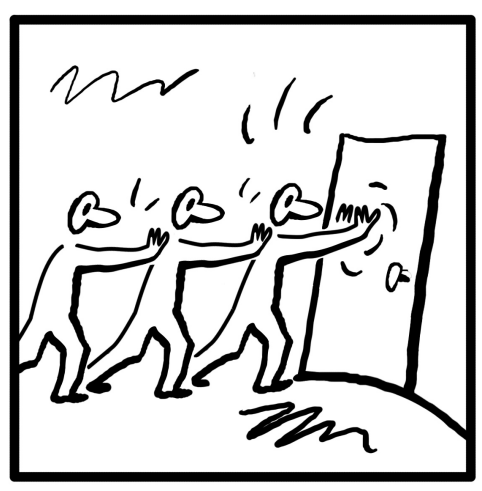

7. MSSM and LED

Figure 16: Searching for supersymmetry and/or large extra dimensions "under a lamp post."

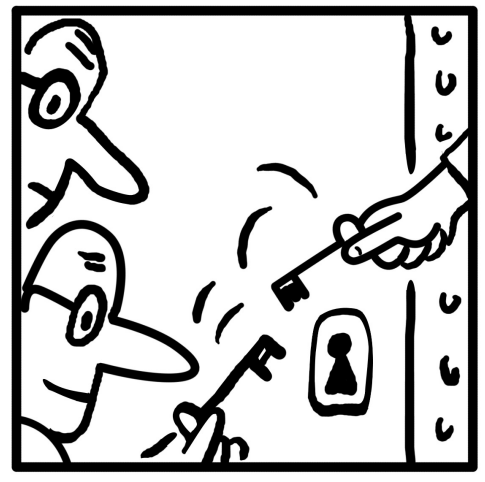

7. MSSM and LED

Figure 17: The search is continued in a different way. 


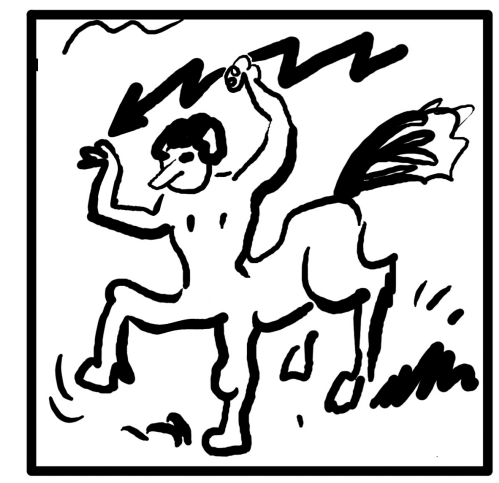

8. EXOTICS (NOTHING SACRED)

Figure 18: All things "beyond" are highly appreciated. If we only new what exactly to look for ...

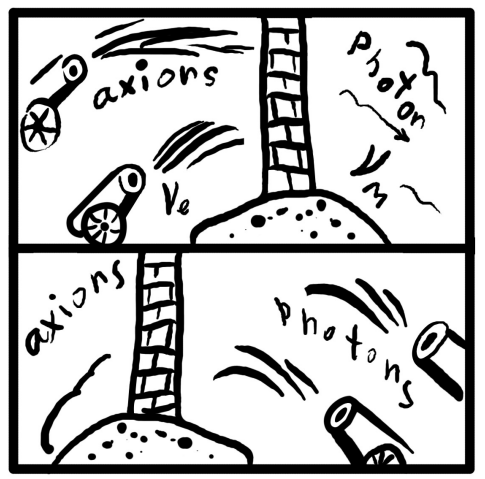

9. PHOTON / AXIONS

Figure 19: Axion physics 


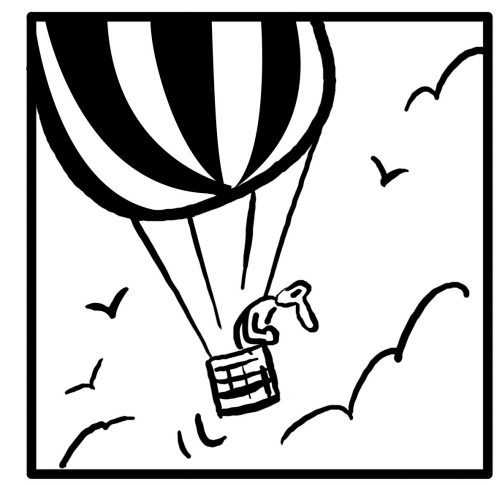

10. QUANTUM GRAVITY DISSENTERS

Figure 20: The ever-lasting passion for quantum gravity 